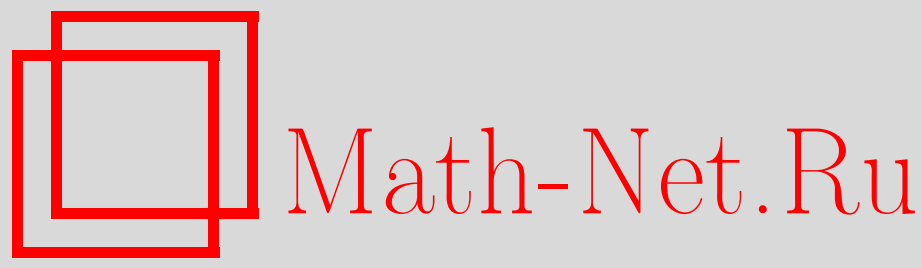

К. В. Жуковский, Решение дифференциальных уравнений эволюционного типа и физических задач с использованием операторного метода, ТМФ, 2017, том 190, номер 1, 58-77

DOI: https://doi.org/10.4213/tmf9144

Использование Общероссийского математического портала Math-Net.Ru подразумевает, что вы прочитали и согласны с пользовательским соглашением http://www . mathnet.ru/rus/agreement

Параметры загрузки:

IP : 54.205 .225 .156

26 апреля 2023 г., 18:02:08

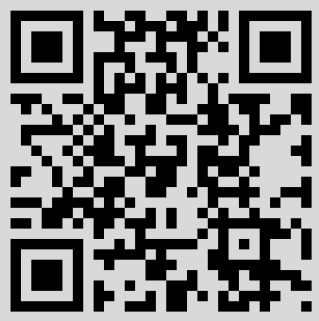




\title{
ФИЗИКА
}

Том 190, № 1

январь, 2017

\section{РЕШЕНИЕ ДИФФЕРЕНЦИАЛЬНЫХ УРАВНЕНИЙ ЭВОЛЮЦИОННОГО ТИПА И ФИЗИЧЕСКИХ ЗАДАЧ С ИСПОЛЬЗОВАНИЕМ ОПЕРАТОРНОГО МЕТОДА}

\begin{abstract}
Представлен общий операторный метод решения широкого круга задач, описываемых некоторыми классами дифференциальных уравнений, на основе развитой техники оператора обратной производной. Сконструированы и применены обратные дифференциальные операторы для решения ряда дифференциальных уравнений. Получены операторные тождества с участием оператора обратной производной, интегральных преобразований и обобщенных форм ортогональных полиномов и специальных функций. Приведены примеры построения решений уравнений, содержащих линейные и квадратичные формы от пары операторов, удовлетворяющих соотношениям типа Гейзенберга, и решения различных модификаций уравнений в частных производных типа теплопроводности Фурье, Фоккера-Планка, Блэка-Шоулза и др. с помощью операторного метода. Продемонстрировано применение операторной техники для решения ряда физических задач, связанных с движением зарядов в рамках квантовой механики, распространением тепла и динамикой пучков в ускорителях.
\end{abstract}

Ключевые слова: обратный оператор, экспоненциальный оператор, обратная производная, дифференциальное уравнение, полиномы Лагерра и Эрмита, специальные функции.

DOI: $10.4213 /$ tmf9144

\section{1. ВВЕДЕНИЕ}

Дифференциальные уравнения (ДУ) являются важнейшим математическим аппаратом для описания широкого спектра физических процессов. Их изучение само по себе представляет серьезную математическую задачу, важность которой трудно переоценить в свете физических приложений получаемых решений. Применение компьютерных методов и развитие вычислительной техники в XXI веке сильно упростило получение решений ДУ. Особую ценность представляют точные аналитические решения, которые позволяют провести глубокий и всесторонний анализ

*Физический факультет, Московский государственный университет имени М. В. Ломоносова, Москва, Россия. E-mail: zhukovsk@physics.msu.ru 
как математической стороны проблемы, так и физических особенностей моделируемого процесса. Получение точных аналитических решений - сложная и далеко не всегда разрешимая задача даже с применением современных компьютерных программ аналитических вычислений, появившихся в последнее десятилетие. Аналитическому описанию поведения физических систем всегда уделяется много внимания. Об этом говорят примеры недавних исследований явлений нелинейной электродинамики, спектров и динамики атомов и особенностей движения и излучения зарядов в магнитных полях [1]-[10], переноса тепла, не подчиняющегося закону Фурье [11]-[14], финансовых и даже некоторых биологических моделей [15], которые позволили частично или полностью аналитически описать поведение сложных систем.

Для решения уравнения Фоккера-Планка в статье [16] были применены интегральные преобразования, и результаты представлены в виде сходящихся рядов. Для точного аналитического решения линейных дифференциальных уравнений полезен метод обратных дифференциальных операторов [17]-[22]. При этом используются расширенные формы ортогональных полиномов Эрмита и Лагерра [23] со многими индексами и переменными [24], [25] и их операторные определения [26]. Интегральные соотношения для тензорных полиномов исследовались в работе [27].

В настоящей работе развитая автором операторная техника решения дифференциальных уравнений обобщается на более сложные уравнения типа теплопроводности, Блэка-Шоулза и Фоккера-Планка с дополнительными слагаемыми. Мы покажем, каким образом использование обратных дифференциальных операторов в сочетании с интегральными преобразованиями и операторными определениями расширенных форм ортогональных полиномов дают возможность быстро решать сравнительно сложные задачи.

\section{2. ОПЕРАТОРНОЕ РЕШЕНИЕ ОБЫКНОВЕННЫХ ДИФФЕРЕНЦИАЛЬНЫХ УРАВНЕНИЙ НЕЦЕЛОГО ПОРЯДКА}

Традиционный подход к решению неоднородных дифференциальных уравнений заключается в использовании функции Грина. Другой подход заключается в использовании обратных дифференциальных операторов, в том числе нецелого порядка. Рассмотрим следующее ДУ:

$$
\left(\beta^{2}-\left(d_{x}+\alpha\right)^{2}\right)^{\nu} F(x)=f(x),
$$

где $\alpha$ и $\beta$ - константы, а $\nu$ является произвольным действительным параметром. Для нахождения частного интеграла применим известное операторное тождество [26]

$$
\hat{q}^{-\nu}=\frac{1}{\Gamma(\nu)} \int_{0}^{\infty} e^{-\hat{q} t} t^{\nu-1} d t, \quad \min \{\operatorname{Re}(q), \operatorname{Re}(\nu)\}>0,
$$

интегральное представление (см. книгу [28])

$$
e^{\hat{p}^{2}}=\frac{1}{\sqrt{\pi}} \int_{-\infty}^{\infty} e^{-\xi^{2}+2 \xi \hat{p}} d \xi=\frac{1}{\sqrt{\pi}} \int_{-\infty}^{\infty} e^{-\xi^{2}-2 \xi \hat{p}} d \xi,
$$

где в нашем случае $\hat{p}=\sqrt{t} \widetilde{D}$ и операторы сдвига и теплопроводности имеют вид [29]

$$
\widehat{\Theta} f(x)=e^{\eta\left(\partial_{x}+\alpha\right)} f(x)=e^{\eta \alpha} f(x+\eta), \quad \widehat{S}=e^{t \partial_{x}^{2}}
$$


соответственно. Действие последнего оператора легко представить в виде преобразования Гаусса-Вейерштрасса. В рамках операторного подхода целесообразно задавать полиномы с помощью операторных соотношений (см. книгу [29] и статьи [19], [30]). Так, для обобщенных форм полиномов Эрмита и Лагерра $H_{n}(x, y)$ и $L_{n}(x, y)$ имеем соответственно равенства

$$
H_{n}(x, y)=e^{y \partial^{2} / \partial x^{2}} x^{n}, \quad L_{n}(x, y)=e^{-y \partial_{x} x \partial_{x}} \frac{(-x)^{n}}{n !}
$$

Производная Лагерра ${ }_{L} D_{x} \equiv \partial_{x} x \partial_{x}=\partial / \partial D_{x}^{-1}[29]$ составляет коммутатор с обратной производной $D_{x}^{-1}:\left[{ }_{\mathrm{L}} D_{x}, D_{x}^{-1}\right]=-1$, где

$$
D_{x}^{-n} f(x)=\frac{1}{(n-1) !} \int_{0}^{x}(x-\xi)^{n-1} f(\xi) d \xi, \quad n \in N=\{1,2, \ldots\}
$$

(см. работу [19]).

Продемонстрируем применение обратных дифференциальных операторов и ортогональных полиномов для решения дифференциальных уравнений. Рассмотрим, например, ОДУ нецелого порядка (1) с мономом $f(x)=x^{k}$. Используем тривиальное операторное соотношение для сдвига обратного оператора

$$
\left(\psi\left(\partial_{x}+\alpha\right)\right)^{-1} f(x)=e^{-\alpha x}\left(\psi\left(\partial_{x}\right)\right)^{-1} e^{\alpha x} f(x)
$$

и соотношение $e^{y \partial_{x}^{2}} x^{k} e^{\alpha x}=e^{\left(\alpha x+\alpha^{2} y\right)} H_{k}(x+2 \alpha y, y)$ [21]. Тогда частный интеграл для уравнения (1) с начальным условием $f(x)=x^{k}$ можно представить в следующем виде:

$$
F(x)=\left(\beta^{2}-\left(d_{x}+\alpha\right)^{2}\right)^{-\nu} x^{k}=\frac{1}{\Gamma(\nu)} \int_{0}^{\infty} e^{-t\left(\beta^{2}-\alpha^{2}\right)} t^{\nu-1} H_{k}(x+2 \alpha t, t) d t
$$

Подынтегральное выражение содержит полиномы Эрмита со сдвинутым аргументом. Отметим, что решение (7) уравнения (1) представимо в виде ряда от свертки $\phi(x)=\Phi(x) * f(x)$ с ядром $\Phi(x)=x^{n}$ и с весом, заданным полиномами Эрмита:

$$
\begin{gathered}
F(x)=\sum_{n=0}^{\infty} \phi(x) C(\nu, \alpha, \beta) \\
\phi(x)=\int_{-\infty}^{\infty} \Phi(x-\eta) f(\eta) d \eta \equiv \Phi(x) * f(x) \\
\Phi(x-\eta)=(\eta-x)^{n}, \quad \Phi(x)=(-x)^{n}, \\
C(\nu, \alpha, \beta)=\frac{1}{\sqrt{\pi} \Gamma(\nu)} \int_{0}^{\infty} \tau^{2(\nu-1)} e^{-\beta^{2} \tau^{2}} \frac{1}{n !} H_{n}\left(\alpha,-\frac{1}{4 \tau^{2}}\right) d \tau .
\end{gathered}
$$

Более того, с учетом того, что выражение для производящей экспоненты имеет вид $e^{x t+y t^{2}}=\sum_{n=0}^{\infty}\left(t^{n} / n !\right) H_{n}(x, y)$, легко показать, что решение уравнения (1) дается интегралом с весом от следующей свертки с ядром распределения вероятности типа 
Гаycca $\Omega(x, \tau)$ :

$$
\begin{gathered}
F(x)=\frac{1}{\sqrt{\pi} \Gamma(\nu)} \int_{0}^{\infty} \tau^{2(\nu-1)} e^{-\beta^{2} \tau^{2}} \varpi(x, \tau) d \tau \\
\varpi(x, \tau)=\int_{-\infty}^{\infty} \Omega(x-\eta, \tau) f(\eta) d \eta \equiv \Omega(x, \tau) * f(x), \\
\Omega(x-\eta, \tau)=\exp \left(\alpha(\eta-x)-\frac{(\eta-x)^{2}}{4 \tau^{2}}\right), \quad \Omega(x, \tau)=\exp \left(-\alpha x-\frac{x^{2}}{4 \tau^{2}}\right) .
\end{gathered}
$$

С учетом того, что

$$
\int_{0}^{\infty} \tau^{2(\nu-1)} \exp \left(-(\beta \tau)^{2}-\frac{(x-\eta)^{2}}{4 \tau^{2}}\right) d \tau=\left(\frac{|x-\eta|}{2 \beta}\right)^{\nu-1 / 2} K_{\nu-1 / 2}(\beta|x-\eta|),
$$

где $K_{n}(x)$ - функция Макдональда, решение принимает следующий вид:

$$
F(x)=\frac{1}{\sqrt{\pi} \Gamma(\nu)} \int_{-\infty}^{\infty}\left(\frac{|x-\eta|}{2 \beta}\right)^{\nu-1 / 2} K_{\nu-1 / 2}(\beta|x-\eta|) e^{-\alpha(x-\eta)} f(\eta) d \eta .
$$

Таким образом, мы получили решение ОДУ нецелого порядка (1) операторным методом в виде свертки:

$$
\begin{gathered}
F(x)=\frac{1}{\sqrt{\pi} \Gamma(\nu)} \int_{-\infty}^{\infty} \chi(x-\eta) f(\eta) d \eta \\
\chi(x-\eta)=\left(\frac{|x-\eta|}{2 \beta}\right)^{\nu-1 / 2} K_{\nu-1 / 2}(\beta|x-\eta|) e^{-\alpha(x-\eta)}
\end{gathered}
$$

с ядром $\chi$. Его можно записать сокращенно как

$$
F(x)=\frac{1}{\sqrt{\pi} \Gamma(\nu)} \chi * f, \quad \chi=\left(\frac{|x|}{2 \beta}\right)^{\nu-1 / 2} K_{\nu-1 / 2}(\beta|x|) e^{-\alpha x}
$$

Метод обратных дифференциальных и экспоненциальных операторов находит многочисленные применения для решения физических задач. Некоторые из них рассматривались в работах [14], [17], [19]. Прежде чем обратиться к примерам, отметим, что соотношение (6), несмотря на его тривиальность, позволяет существенно продвинуться в решении операторным методом некоторых дифференциальных уравнений. Так, в соответствии с соотношением (6) мы можем записать для уравнения $f(x, t)=\psi\left(\partial_{x}+\kappa\right) F(x, t)$ следующее равенство: $e^{\kappa x} F(x, t)=\psi^{-1}\left(\partial_{x}\right) e^{\kappa x} f(x, t)$. Тогда, например, для функции $f(x, t)=\partial_{t} F(x, t)$ имеем $\psi\left(\partial_{x}\right) e^{\kappa x} F(x, t)=\partial_{t} e^{\kappa x} F(x, t)$. Введя обозначение $G(x, t)=e^{\kappa x} F(x, t)$, получаем уравнение $\psi\left(\partial_{x}\right) G(x, t)=\partial_{t} G(x, t)$ с начальным условием $g(x)=G(x, 0)=e^{\kappa x} F(x, 0)=e^{\kappa x} f(x)$. Таким образом, чтобы получить искомое решение $F(x, t)=e^{-\kappa x} G(x, t)$ уравнения $\psi\left(\partial_{x}+\kappa\right) F(x, t)=f(x, t)$ с оператором $\psi\left(\partial_{x}+\kappa\right)$ и с начальным условием $F(x, 0)=f(x)$, нужно решить уравнение с оператором $\psi\left(\partial_{x}\right)$ на функцию $G(x, t)$ с соответствующим начальным условием $g(x)=e^{\kappa x} f(x)$. 


\section{3. ОПЕРАТОРНОЕ РЕШЕНИЕ УРАВНЕНИЙ ТИПА БЛЭКА-ШОУЛЗА}

Продемонстрируем обозначенную выше технику решением следующего дифференциального уравнения типа Блэка-Шоулза:

$$
\frac{1}{\rho} \frac{\partial}{\partial t} F(x, t)=\left[x^{2} \frac{\partial^{2}}{\partial x^{2}}+\left(2 \alpha x^{2}+\lambda x\right) \frac{\partial}{\partial x}+(\alpha x)^{2}-\mu\right] F(x, t), \quad f(x)=F(x, 0),
$$

где $\alpha, \rho, \lambda$ и $\mu$ - постоянные коэффициенты и $f(x)=F(x, 0)$ - функция начального условия. Легко заметить, что (13) является уравнением со сдвинутой производной, и оно сводится к уравнению вида

$$
\frac{1}{\rho} \frac{\partial}{\partial t} G(x, t)=x^{2} \frac{\partial^{2}}{\partial x^{2}} G(x, t)+\lambda x \frac{\partial}{\partial x} G(x, t)-\mu G(x, t), \quad g(x)=G(x, 0),
$$

путем замены $\partial_{x} \rightarrow \partial_{x}+\alpha$. Тогда решение уравнения (13) можно записать через решение $G(x, t)$ уравнения (14) с начальным условием $g(x)=G(x, 0)=e^{\alpha x} F(x, 0)$ :

$$
F(x, t)=e^{-\alpha x} G(x, t), \quad g(x)=G(x, 0)=e^{\alpha x} F(x, 0) .
$$

Уравнение (14) в свою очередь было решено операторным методом (см. статью [19]). Выберем начальное условие для уравнения типа Блэка-Шоулза со сдвигом переменной (13) в виде $f(x)=e^{-a x} x^{n}$. Тогда $g(x)=x^{n}$ и с учетом равенства (см. [19])

$$
G(x, t)=\frac{e^{-\rho \varepsilon t}}{\sqrt{\pi}} \int_{-\infty}^{\infty} e^{-\sigma^{2}+\sigma \gamma \lambda / 2 \rho} g\left(x e^{\sigma \gamma}\right) d \sigma
$$

искомое решение уравнения (13) принимает простой вид

$$
\left.F(x, t)\right|_{f(x)=e^{-a x} x^{n}}=e^{-\alpha x} x^{n} e^{\rho t\left(n^{2}+\lambda n-\mu\right)} .
$$

Другим примером является следующее обобщение дифференциального уравнения типа Блэка-Шоулза с производной Лагерра ${ }_{\mathrm{L}} D_{x}$ :

$$
\frac{1}{\rho} \frac{\partial}{\partial t} A(x, t)=\left(\partial_{x} x \partial_{x}\right)^{2} A(x, t)+\lambda\left(\partial_{x} x \partial_{x}\right) A(x, t)-\mu A(x, t), \quad g(x)=A(x, 0),
$$

где $\rho, \lambda$ и $\mu$ - постоянные коэффициенты. Отметим, что уравнение (17) по существу является обобщением двух уравнений: диффузии и теплопроводности Лагерра, рассмотренных ранее в работах [19] и [25]. Следуя [19], запишем решение (17) в виде $A(x, t)=e^{\rho t\left(\left({ }_{\mathrm{L}} D_{x}+\lambda / 2\right)^{2}-\mu-(\lambda / 2)^{2}\right)} g(x)$ и с помощью операторного соотношения $(3)$ получим следующее решение для $A(x, t)$ :

$$
A(x, t)=\frac{e^{-\rho t\left(\mu+(\lambda / 2)^{2}\right)}}{\sqrt{\pi}} \int_{-\infty}^{\infty} e^{-\sigma\left(\sigma+\sqrt{\rho t}\left(\lambda-2_{\mathrm{L}} D_{x}\right)\right)} g(x) d \sigma .
$$

Выберем начальную функцию в виде $g(x)=(-x)^{n} / n$ !. С учетом операторного определения полиномов Лагерра (5) имеем

$$
A(x, t)=\frac{e^{-\varepsilon \rho t / 4}}{\sqrt{\pi}} \int_{-\infty}^{\infty} e^{-\sigma(\sigma+\sqrt{\rho t} \lambda)} L_{n}(x, 2 \sigma \sqrt{\rho t}) d \sigma .
$$


Интегрируя по $d \sigma$, получаем следующее решение обобщенного уравнения БлэкаШоулза с производной Лагерра (17) с начальным условием $g(x)=(-x)^{n} / n$ !:

$$
\begin{aligned}
A(x, t)= & \frac{e^{-\rho t \mu}}{\sqrt{\pi}} n ! \sum_{r=0}^{n} \frac{(-x)^{r}(2 \sqrt{\rho t})^{n-r}}{(n-r) !(r !)^{2}} \times \\
& \times\left\{\frac{e^{i(n-r) \pi}+1}{2} \Gamma\left(\frac{1+n-r}{2}\right){ }_{1} F_{1}\left(-\frac{n-r}{2}, \frac{1}{2},-\left(\frac{\lambda \sqrt{\rho t}}{2}\right)^{2}\right)+\right. \\
& \left.+\frac{e^{i(n-r) \pi}-1}{2} \lambda \sqrt{\rho t} \Gamma\left(1+\frac{n-r}{2}\right){ }_{1} F_{1}\left(\frac{1-(n-r)}{2}, \frac{3}{2},-\left(\frac{\lambda \sqrt{\rho t}}{2}\right)^{2}\right)\right\},
\end{aligned}
$$

где $\Gamma$ - гамма-функция, ${ }_{1} F_{1}$ - гипергеометрическая функция. Очевидно, что решение для начального условия в виде полинома по $x$ соответствует сумме от выражения (20), а для функции начального условия, заданной как ряд по полиномам Лагерра $g(x)=\sum_{n} a_{n} L_{n}(x)$, решение записывается как ряд следующего вида:

$$
A(x, t)=\frac{e^{-\varepsilon \rho t / 4}}{\sqrt{\pi}} \sum_{n} a_{n} \int_{-\infty}^{\infty} e^{-\sigma(\sigma+\lambda \sqrt{\rho t})} L_{n}(x, 2 \sigma \sqrt{\rho t}+1) d \sigma .
$$

Введем образ $\varphi(x)=\int_{0}^{\infty} e^{-\kappa} g(x \kappa) d \kappa$ функции $g(x)=\varphi\left(D_{x}^{-1}\right)\{\mathbf{1}\}$ подобно тому, как описано в статье [19]. Тогда решение уравнения (17) с учетом равенств $\left[{ }_{\mathrm{L}} D_{x}, D_{x}^{-1}\right]=-1$ и $e^{-t \partial / \partial D_{x}^{-1}} \varphi\left(D_{x}^{-1}\right)=e^{-t \partial / \partial D_{x}^{-1}} g(x)=\varphi\left(D_{x}^{-1}-t\right)\{\mathbf{1}\}$ можно выразить через интеграл от оператора обратной производной $D_{x}^{-1}$ при условии его сходимости:

$$
A(x, t)=\frac{e^{-\varepsilon \rho t}}{\sqrt{\pi}} \int_{-\infty}^{\infty} e^{-\sigma(\sigma+\alpha \lambda)} g(x, t) d \sigma,
$$

где

$$
g(x, t)=\varphi\left(D_{x}^{-1}-2 \sigma \alpha\right)\{\mathbf{1}\}=e^{-2 \sigma \alpha \partial / \partial D_{x}^{-1}} \varphi\left(D_{x}^{-1}\right)\{\mathbf{1}\} .
$$

Например, для $g(x)=W_{0}\left(-x^{2}, 2\right)$, где

$$
W_{n}(x, m)=\sum_{r=0}^{\infty} \frac{x^{r}}{r !(m r+n) !}, \quad m \in \mathbb{N}, \quad n \in \mathbb{N}_{0},
$$

есть частный случай функции Бесселя-Райта [29], ее образом оказывается функция $\varphi(x)=e^{-x^{2}}$, и, применяя операторное соотношение $(3)$, получаем в соответствии с (23) (см. статью [19]) формулу

$$
g(x, t)=\frac{1}{\sqrt{\pi}} \int_{-\infty}^{\infty} e^{-\xi^{2}+4 i \sigma \alpha \xi} C_{0}(2 i \xi x) d \xi
$$

где $C_{n}(x)$ - функция Бесселя-Трикоми [31], связанная с функцией Бесселя-Райта и с функциями Бесселя:

$$
C_{n}(x)=W_{n}(-x, 1)=x^{-n / 2} J_{n}(2 \sqrt{x})=\sum_{r=0}^{\infty} \frac{(-x)^{r}}{r !(r+n) !}, \quad n \in \mathbb{N}_{0} .
$$

Некоторые модификации уравнения Блэка-Шоулза исследовались операторным методом в статьях [32] и [33]. 


\section{4. ОПЕРАТОРНОЕ РЕШЕНИЕ РАСШИРЕННОГО УРАВНЕНИЯ ТЕПЛОПРОВОДНОСТИ ФУРЬЕ И УРАВНЕНИЙ ТИПА ШРЕДИНГЕРА}

Динамика заряда, проходящего под потенциальным барьером в электростатическом поле, описывается обобщенным уравнением теплопроводности с линейным члеHOM

$$
\partial_{t} G(x, t)=\alpha \partial_{x}^{2} G(x, t)+\beta x G(x, t), \quad G(x, 0)=g(x),
$$

и представляет по существу уравнение Шредингера с мнимым временем. Соответствующий этому евклидов подход в квантовой механике подробно описан, например, в книге [34]. Явление туннелирования частицы в области, где ее энергия ниже, чем потенциальная энергия барьера, представляется важным также при исследовании вакуумных эффектов квантовой хромодинамики. В контексте распространения тепла второе слагаемое в правой части (25) описывает излучение в окружающую среду с небольшой разницей температур через теплоизолирующую прокладку с линейно меняющейся по координате толщиной. Уравнение (25) исследовалось в работах [17], [18] и [20]; было получено следующее решение в виде последовательного действия операторов $\widehat{\bar{S}}$ и $\widehat{\bar{\Theta}}(4)$ на функцию начального условия $G(x, 0)=g(x)$ :

$$
\begin{aligned}
G(x, t) & =e^{\Phi(x, t ; \alpha, \beta)} \widehat{\Theta} \widehat{S} g(x)=e^{\Phi(x, t ; \alpha, \beta)} g\left(x+\alpha \beta t^{2}, t\right)= \\
& =\frac{e^{\Phi(x, t ; \alpha, \beta)}}{2 \sqrt{\pi \alpha t}} \int_{-\infty}^{\infty} e^{-\left(x+\alpha \beta t^{2}-\xi\right)^{2} / 4 t \alpha} g(\xi) d \xi
\end{aligned}
$$

где $\widehat{\Theta}=e^{\alpha \beta t^{2} \partial_{x}}, \widehat{S}=e^{\alpha t \partial_{x}^{2}}$ и $\Phi(x, t ; \alpha, \beta)=\alpha \beta^{2} t^{3} / 3+\beta t x$. Для практического изучения распространения тепла особую значимость имеют решения, описывающие эволюцию импульсов, так как это является устоявшимся экспериментальным методом исследования теплопроводности веществ. Рассмотрим уравнение (25) с начальным условием $g(x)=x^{k} e^{\delta x}$. Оно моделирует асимметричный импульс при $\delta<0$, а аппроксимация экспериментальных данных рядом $\sum_{k, \delta} x^{k} e^{\delta x}$ дает возможность исследовать эволюцию практически любых импульсов аналитически. В соответствии с (26) и (4) получаем следующее решение (25) при $g(x)=x^{k} e^{\delta x}$ :

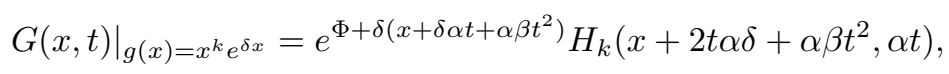

а для $g(x)=\sum_{k, \delta} x^{k} e^{\delta x}$ имеем $G(x, t)=e^{\Phi} \sum_{k, \delta} e^{\delta\left(x+\delta \alpha t+\alpha \beta t^{2}\right)} H_{k}\left(x+2 t \alpha \delta+\alpha \beta t^{2}, \alpha t\right)$.

Рассмотрим обобщение уравнения (25), имеющее вид

$$
\partial_{t} F(x, t)=\alpha \partial_{x}^{2} F(x, t)+\varsigma \partial_{x} F(x, t)+\beta x F(x, t)+\gamma F(x, t), F(x, 0)=f(x) .
$$

Следуя общей логике решения уравнений со сдвинутой производной (см. начало данного раздела) и выделяя квадрат оператора $\partial_{x}+\kappa$, где $\kappa=\varsigma / 2 \alpha$, получаем решение уравнения (28)

$$
F(x, t)=e^{t\left(\gamma-\alpha \kappa^{2}-\kappa x\right)} G(x, t)
$$

где функция $G(x, t)$ удовлетворяет уравнению $(25)$ с начальным условием $g(x)=e^{\kappa x} f(x)$. Выберем начальное условие для (28) в виде монома $f(x)=x^{k}$. Тогда $g(x)=x^{k} e^{\kappa x}$ 
и, подставляя решение (27) уравнения (25) в (29), немедленно получаем искомое решение уравнения (28), записанное через полиномы Эрмита:

$$
\left.F(x, t)\right|_{f(x)=x^{k}}=\exp \left(\frac{\alpha \beta^{2} t^{3}}{3}+\frac{\varsigma \beta t^{2}}{2}+\beta t x+t \gamma\right) H_{k}\left(x+\varsigma t+\alpha \beta t^{2}, \alpha t\right) .
$$

Рассмотрим теперь следующее двумерное уравнение теплопроводности:

$$
\partial_{t} F(x, y, t)=\left\{\left(\alpha \partial_{x}^{2}+\varepsilon \partial_{x} \partial_{y}+\gamma \partial_{y}^{2}\right)+b x+c y\right\} F(x, y, t), \quad \min (\alpha, \varepsilon, \gamma)>0,
$$

с начальным условием $F(x, y, 0)=f(x, y)$, которое также описывает двумерную динамику заряда в электрическом поле при прохождении под потенциальным барьером. Операторное решение уравнения (31) можно получить по аналогии с решением одномерного уравнения (25) (см. статьи [17], [20]) или с помощью формулы Бейкера-Кемпбелла-Хаусдорфа. Оно представляет собой последовательное действие двумерного аналога $\widehat{S}$ - оператора теплопроводности

$$
\widehat{\mathrm{E}}=e^{t\left(\alpha \partial_{x}^{2}+\varepsilon \partial_{x} \partial_{y}+\gamma \partial_{y}^{2}\right)}
$$

на начальное условие: $\widehat{\mathrm{E}} f(x, y)=f(x, y, t)$, и действие операторов координатного сдвига $\widehat{\Theta}_{x}=e^{t^{2}(\alpha b+\varepsilon c / 2) \partial_{x}}$ и $\widehat{\Theta}_{y}=e^{t^{2}(\gamma c+\varepsilon b / 2) \partial_{y}}$ на $f(x, y, t)$. В результате получаем решение

$$
F(x, y, t)=e^{\Psi} \widehat{\Theta}_{x} \widehat{\Theta}_{y} \widehat{\mathrm{E}} f(x, y) \propto f\left(x+t^{2}\left(\alpha b+\frac{\varepsilon c}{2}\right), y+t^{2}\left(\gamma c+\frac{\varepsilon b}{2}\right), t\right),
$$

где $\Psi=\left(\alpha b^{2}+\gamma c^{2}+\varepsilon b c\right) t^{3} / 3+t(b x+c y)-$ фаза. Действие оператора $\widehat{\mathrm{E}}$ имеет вид двукратного интеграла типа Гаусса (см. работу [22]). При $\varepsilon=0$ в (31) вместо оператора теплопроводности $\widehat{\mathrm{E}}$ имеем произведение операторов теплопроводности (4) $\widehat{S}_{x} \widehat{S}_{y}$ по каждой из координат, так что решение принимает следующий вид:

$$
F(x, y, t ; \varepsilon=0)=e^{\Psi} \widehat{\Theta}_{x} \widehat{\Theta}_{y} \widehat{S}_{x} \widehat{S}_{y} f(x, y) \propto f\left(x+t^{2} \alpha b, y+t^{2} \gamma c, t\right) .
$$

Операторное определение (32) позволяет непосредственно получить решение уравнения (31), например, для степенной функции начального условия $f(x, y)=x^{m} y^{n}$. Действие оператора теплопроводности $\widehat{\mathrm{E}}$ на $f$ дает выражение

$$
\widehat{\mathrm{E}}\left\{x^{m} y^{n}\right\}=H_{m, n}(x, t \alpha, y, t \gamma \mid t \varepsilon),
$$

где $H_{m, n}(x, t \alpha, y, t \gamma \mid t \varepsilon)$ - полиномы Эрмита трех переменных и параметров с двумя индексами (см. статьи [19], [22], [26]). Последующий сдвиг операторами $\widehat{\Theta}_{x} \widehat{\Theta}_{y}$ приводит к следующему решению двумерного уравнения теплопроводности (31) c $f(x, y)=x^{m} y^{n}$ :

$$
F(x, t)=e^{\Psi} H_{m, n}\left(x+t^{2}\left(\alpha b+\frac{\beta c}{2}\right), t \alpha ; y+t^{2}\left(\gamma c+\frac{\beta b}{2}\right), t \gamma \mid t \varepsilon\right) .
$$

Очевидно, что полученное решение (36) двумерного уравнения теплопроводности (31) является непосредственным обобщением решения (27) с $\delta=0$ для одномерного аналога (25) уравнения (31). Дальнейшее обобщение на трехмерный случай не составляет труда. 


\section{5. РАСПРОСТРАНЕНИЕ $\delta$-ИМПУЛЬСА В РАМКАХ ТЕПЛОПРОВОДНОСТИ ФУРЬЕ}

Экспериментальное значение теплопроводности различных материалов обычно определяется при помощи замеров тепловых импульсов [35]. При этом измеряется характерное время релаксации [36]. В качестве начального импульса часто используют сверхкороткий точечный импульс лазера. Моделью последнего является $\delta$-функция Дирака. Проведем математическое моделирование распространения такого импульса в уравнении теплопроводности Фурье в двумерном случае $f(x, y)=\delta(x, y)$. Действие оператора $\widehat{S}$ на $\delta$-функцию дает распределение типа Гаyсca:

$$
f(x, y, t)=\widehat{S}_{x} \widehat{S}_{y} \delta(x, y)=\frac{\exp \left[-\left(x^{2} / \alpha+y^{2} / \gamma\right) / 4 t\right]}{4 \pi t \sqrt{\alpha \gamma}},
$$

а операторы сдвига $\widehat{\Theta}_{x, y}$ при наличии линейных членов в уравнении теплопроводности Фурье приводят к следующему решению с начальной функцией $f(x, y)=\delta(x, y)$ :

$$
\begin{aligned}
\left.F(x, y, t)\right|_{f=\delta(x, y)} & \equiv \chi(x, y, t)=e^{\Psi} \widehat{\Theta}_{x} \widehat{\Theta}_{y} \widehat{S}_{x} \widehat{S}_{y} \delta(x, y)= \\
& =e^{\Psi} \frac{\exp \left(-\left[\left(x+t^{2} \alpha b\right)^{2} / \alpha+\left(y+t^{2} \gamma c\right)^{2} / \gamma\right] / 4 t\right)}{4 \pi t \sqrt{\alpha \gamma}},
\end{aligned}
$$

а его одномерный аналог в случае уравнения (25) при $f(x)=\delta(x)$ имеет вид

$$
\left.F(x, t)\right|_{f=\delta(x)} \equiv \chi(x, t)=e^{\Phi} \frac{\exp \left(-\left(x+\alpha \beta t^{2}\right)^{2} / 4 t \alpha\right)}{2 \sqrt{\pi t \alpha}},
$$

где $\Phi, \Psi$ были определены выше (см. (26), (33)). Непосредственной подстановкой легко проверить, что полученные выше решения удовлетворяют уравнениям (25), (31).

Найдем теперь решение расширенного уравнения теплопроводности (28) с начальным условием $f(x)=\delta(x)$. Выделим $\partial_{x}+\kappa$, где $\kappa=\varsigma / 2 \alpha$, и воспользуемся результатом (29). Решение для функции $G(x, t)$ с начальным условием $g(x)=e^{\kappa x} \delta(x)$ легко найти из (26):

$$
\begin{aligned}
\left.G(x, t)\right|_{g(x)=e^{\kappa x} \delta(x)} & =\frac{e^{\Phi(x, t ; \alpha, \beta)}}{2 \sqrt{\pi \alpha t}} \int_{-\infty}^{\infty} e^{-\left(x+\alpha \beta t^{2}-\xi\right)^{2} / 4 t \alpha} e^{\kappa \xi} \delta(\xi) d \xi= \\
& =\frac{e^{\Phi(x, t ; \alpha, \beta)}}{2 \sqrt{\pi \alpha t}} e^{-\left(x+\alpha \beta t^{2}\right)^{2} / 4 t \alpha} .
\end{aligned}
$$

Искомое решение уравнения (28) с $f(x)=\delta(x)$ принимает следующий вид:

$$
\left.F(x, t)\right|_{f(x)=\delta(x)}=\exp \left(\frac{1}{3} \alpha \beta^{2} t^{3}+t\left(\beta x+\gamma-\frac{\varsigma^{2}}{4 \alpha}\right)-\frac{\varsigma}{2 \alpha} x\right) \frac{1}{2 \sqrt{\pi \alpha t}} e^{-\left(x+\alpha \beta t^{2}\right)^{2} / 4 t \alpha} .
$$

Отметим, что линейное слагаемое присутствует в уравнениях различных моделей теплопроводности (см., например, статью [11]). Пример решения (41) расширенного уравнения Фурье (28) показывает, что начальная $\delta$-функция затухает, но затем происходит неограниченный экспоненциальный рост решения со сдвигом максимума относительно точки $x=0$, на которую приходится максимум в начальный момент 


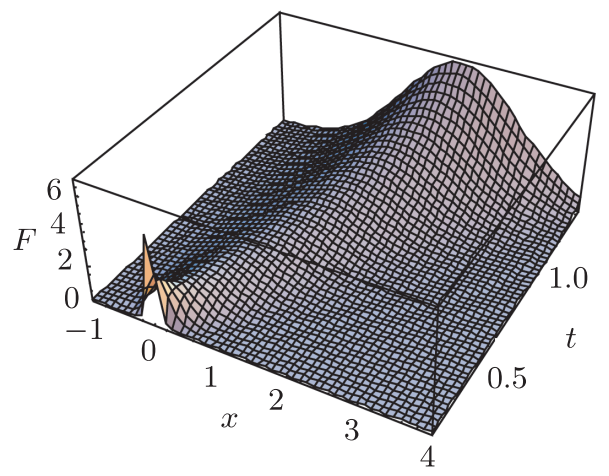

Рис. 1. Эволюция начальной функции $\delta(x)$ в расширенном уравнении теплопроводности Фурье с $\alpha=0.3, \beta=\gamma=-\varsigma=1, t \in[0.01,1.43]$.

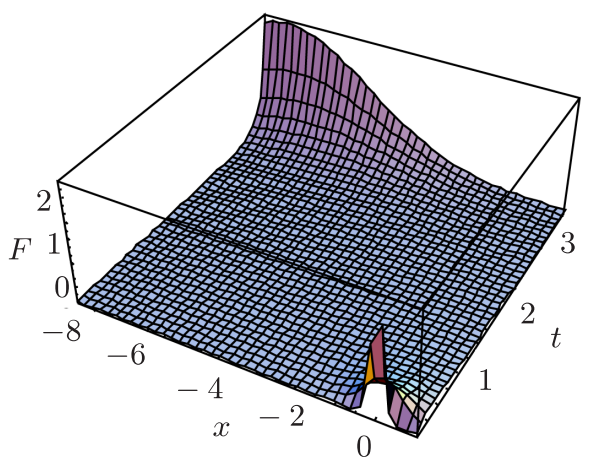

Рис. 2. Эволюция начальной функции $\delta(x)$ в расширенном уравнении теплопроводности Фурье с $\alpha=1, \beta=\gamma=\varsigma=-1, t \in[0.01,3.2]$.

(см. рис. 1 , где $\alpha=0.3, \beta=\gamma=-\varsigma=1$ ). Поведение решения (39) уравнения (25) при $\alpha=0.3, \beta=3$ качественно похоже на показанное на рис. 1 ; график не приводится для краткости. Выбором значений параметров $\alpha, \beta, \gamma, \varsigma$ можно добиться того, что решение практически полностью затухает в широкой области (см. рис. 2). Отметим слабую асимметрию решения (39) относительно точки начального максимума $x=0$ при малых временах $t$ из-за линейного члена $\beta \neq 0$.

Полученное решение (39) обычного уравнения Фурье (25) с $\beta=0$ затухает при $t \rightarrow \infty$ (см. рис. 3). Таким образом, хотя влияние линейного члена в уравнении Фурье (25) при малых временах незначительно (ср. рис. $1-3$ при $t<0.1)$, на больших временах оно становится доминирующим (см. рис. 1 при $t>0.1$ и рис. 2 при $t>2.5)$ !

Более того, решение $F(x, t)$ расширенного уравнения Фурье (28) достигает максимума на границе $t=T$ рассматриваемой области $x \subset[-l, L], t \subset\left[T_{0}, T\right]($ см. рис. 1 , рис. 2), что нарушает принцип максимума, гарантирующий единственность и устойчивость решений. Невыполнение принципа максимума и тот факт, что рост 


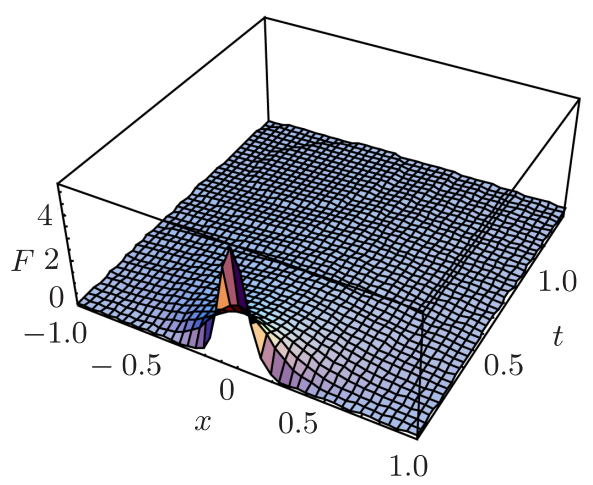

Рис. 3. Эволюция начальной функции $\delta(x)$ в обычном уравнении теплопроводности Фурье с $\alpha=0.3, \beta=\gamma=\varsigma=0, t \in[0.01,1.43]$.

решения со временем неограничен, в контексте переноса тепла не соответствуют второму закону термодинамики, который предполагает, что $\beta=0$.

Возвращаясь к квантовомеханической интерпретации уравнений (25), (31), отметим, что заменой $t \rightarrow-i \tau, \alpha \rightarrow-\alpha, \gamma \rightarrow-\gamma$ в (25), (31) мы получаем обычное уравнение Шредингера для заряда в электростатическом поле, а его решения получаются вышеуказанной заменой в (26), (34) и в (38), (39) соответственно для начальной $\delta$-функции. Полученные решения $F(x, y, t)$ можно рассматривать как амплитуду вероятности перехода частицы из начальной точки $x=0$ при $t=0$ в точку с координатой $x$ за промежуток времени $t>0$. Обратим внимание, что при этом фазы $\Phi, \Psi$ становятся комплексными и не имеют значения с физической точки зрения, так как вероятность определяется величиной $|F(x, y, t)|^{2}$; увеличения этой вероятности со временем за счет фазы подобно тому, как показано на рис. 1, в этом случае не происходит. Под решением $F(x, y, t)$ уравнений $(25),(31)$ также можно понимать концентрацию; при этом каждая частица движется независимо от остальных. Начальная функция $f(x)=\delta(x, y)$ означает, что все частицы находились в точке $(x, y)$ в момент времени $t=0$, и решения уравнений (25), (31) описывают концентрацию частиц через промежуток времени длины $t$. Тогда все результаты с точностью до множителя, равного количеству диффундирующих частиц, относятся к концентрации частиц при условии, что мы пренебрегаем взаимодействием диффундирующих частиц между собой.

Заметим также, что решения (26), (34) уравнений (25), (31) для произвольной функции начального условия представляют собой свертку функции начального условия $f$ с решением для начальной $\delta$-функции Дирака. Таким образом, операторным методом мы получили решение уравнения Шредингера (теплопроводности) для частицы в электростатическом поле, диффундирующей под потенциальным барьером, в виде следующей свертки с ядром $\chi$ :

$$
F(x, y, t)=\left.\int_{-\infty}^{\infty} \chi(x-\eta, y-\rho)\right|_{\substack{t \rightarrow-i \tau, \alpha \rightarrow-\alpha, \gamma \rightarrow-\gamma}} f(\eta, \rho) d \eta d \rho \equiv \chi * f
$$


где ядро $\chi$ типа Гаусса является решением исходного уравнения с $F(x, y, 0)=\delta(x, y)$ и определено формулой (38) с заменой $t \rightarrow-i \tau, \alpha \rightarrow-\alpha, \gamma \rightarrow-\gamma$. Одномерный аналог свертки (42) получается с ядром (39) при той же замене.

Несмотря на то что уравнение Фурье хорошо описывает перенос тепла в однородных недеформируемых твердых телах при нормальных условиях, оно не справедливо при сверхнизких температурах, а также в системах с пониженной размерностью, таких как графен, нановолокна и др., и в материалах с существенной внутренней неоднородностью. Исследование процессов теплопередачи в таких случаях нетривиально и требует специального рассмотрения. Мы обратимся к этому в последующих публикациях.

\section{6. ОПЕРАТОРНОЕ РЕШЕНИЕ УРАВНЕНИЙ ТИПА ФОККЕРА-ПЛАНКА}

Уравнения типа Фоккера-Планка встречаются при моделировании распространения пучков электронов в ускорителях и в ондуляторах. Операторное решение уравнения Фоккера-Планка

$$
\partial_{t} F(x, t)=\alpha \partial_{x}^{2} F(x, t)+\beta x \partial_{x} F(x, t), \quad F(x, 0)=f(x),
$$

проводится по аналогии с решением уравнения Шредингера:

$$
F(x)=\widehat{U} f(x), \quad \widehat{U} f(x)=e^{t \alpha \partial_{x}^{2}+t \beta x \partial_{x}} f(x)=e^{\sigma \partial_{x}^{2}} f\left(e^{\beta t} x\right),
$$

где $\sigma=\left(1-e^{-2 \beta t}\right) \alpha / \beta$ (см., например, статью [17]). В отличие от уравнения Шредингера, в решении уравнений типа Фоккера-Планка начальная функция $f$ преобразуется лишь одним оператором теплопроводности $\widehat{S}$ (cp. (44) с (26)).

Рассмотрим теперь следующее обобщение уравнения Фоккера-Планка:

$$
\partial_{t} F(x, t)=\left\{\alpha \partial_{x}^{2}+(\beta x+2 \alpha \delta) \partial_{x}+\beta \delta x+\gamma\right\} F(x, t) .
$$

Для уравнений типа Фоккера-Планка имеет смысл рассматривать начальное условие $f(x)=e^{-x^{2}}$ типа Гаусса, потому что оно является наиболее распространенным для пучков в ускорителях. Выделив оператор $\partial_{x}+\delta$, получаем, что решение обобщенного уравнения Фоккера-Планка (45) сводится к решению уравнения (43) для функции $G$ с начальным условием $g(x)=G(x, 0)=e^{\alpha x} f(x)=e^{\delta x-x^{2}}$. Решение $G(x, t)$ получается с использованием преобразования Гаусса:

$$
G(x, t)=\frac{1}{\sqrt{2 \pi \rho}} \int_{-\infty}^{\infty} g(\xi) \exp \left[-\left(\frac{e^{\beta t} x-\xi}{\sqrt{2 \rho}}\right)^{2}\right] d \xi
$$

где $\rho(t)=(\alpha / \beta)\left(e^{2 \beta t}-1\right)$. Тогда имеем $F(x, t)=e^{\gamma-\alpha \delta^{2}} e^{-\delta x} G(x, t)$ и окончательно получаем искомое решение уравнения (45) в следующем виде:

$$
\left.F(x, t)\right|_{f(x)=e^{-x^{2}}}=\frac{e^{\gamma-(\delta / 2)^{2}(4 \alpha-1)-\delta x}}{\sqrt{1+2 \rho(t)}} \exp \left(-\frac{\left(e^{\beta t} x-\delta / 2\right)^{2}}{1+2 \rho(t)}\right) .
$$

График решения при $\alpha=1, \beta=4, \gamma=3, \delta=2$ построен на рис. 4. Отметим асимметрию решения за счет отличных от нуля $\gamma$ и $\delta$. Важно также то, что максимум решения достигается внутри рассматриваемой области, что нарушает принцип максимума, гарантирующий единственность и устойчивость решения. 


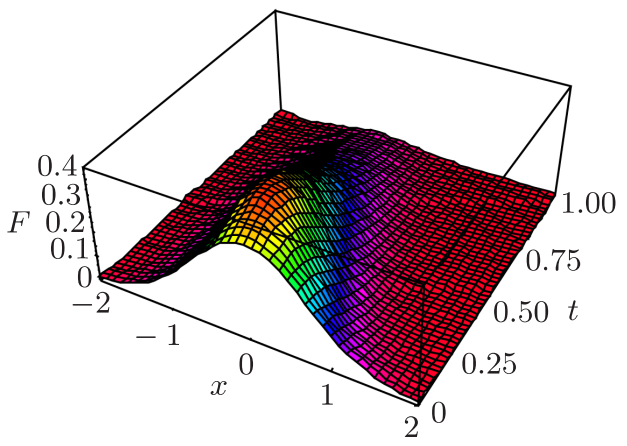

Рис. 4. Пример графика решения уравнения типа Фоккера-Планка $\partial_{t} F(x, t)=$ $\left\{\alpha \partial_{x}^{2}+(\beta x+2 \alpha \delta) \partial_{x}+\beta \delta x+\gamma\right\} F(x, t)$ для $F(x, 0)=e^{-x^{2}}, F(x, \infty)<\infty$ при $\alpha=1, \beta=4, \gamma=3, \delta=2$.

Другая модификация уравнения Фоккера-Планка (43) состоит в добавлении дифференциального оператора второго порядка по времени $\partial_{t}^{2}$ в левую часть этого уравнения:

$$
\left(\frac{\partial^{2}}{\partial t^{2}}+\varepsilon \frac{\partial}{\partial t}\right) F(x, t)=\left(\alpha \frac{\partial^{2}}{\partial x^{2}}+\beta x \partial_{x}\right) F(x, t), \quad \alpha, \beta, \varepsilon=\mathrm{const}
$$

наподобие гиперболического уравнения теплопроводности Каттанео [37], который предложил релаксационную модель теплопроводности, качественно описывающую процесс при низкой температуре за счет фононного механизма передачи тепла в следующем уравнении: $\left(\tau \partial_{t}^{2}+\partial_{t}\right) T=k_{T} \nabla^{2} T$. Исследование этого уравнения остается за рамками настоящей работы; мы вернемся к нему в последующих публикациях. Для решения уравнения (47) используем операторную технику. Так, для уравнений типа

$$
\left(\frac{\partial^{2}}{\partial t^{2}}+\hat{\varepsilon}(x) \frac{\partial}{\partial t}\right) F(x, t)=\widehat{D}(x) F(x, t),
$$

содержащих оператор $\hat{\varepsilon}(x)$, операторное решение с начальным условием $F(x, 0)=f(x)$ и конечным условием $F(x, \infty)=0$ или, по крайней мере, с ограниченным при $t \rightarrow \infty$ решением $F(x, \infty)<\infty$ можно получить с использованием преобразования Лапласа

$$
e^{-t \sqrt{V}}=\frac{t}{2 \sqrt{\pi}} \int_{0}^{\infty} e^{-t^{2} / 4 \xi-\xi V} \frac{d \xi}{\xi \sqrt{\xi}}, \quad t>0
$$

в виде интеграла

$$
F(x, t)=e^{(-t / 2) \hat{\varepsilon}(x)} \frac{t}{4 \sqrt{\pi}} \int_{0}^{\infty} e^{-t^{2} / 16 \xi} e^{-\xi \hat{\varepsilon}^{2}(x)} e^{-4 \xi \widehat{D}(x)} f(x) \frac{d \xi}{\xi \sqrt{\xi}}
$$

при условии, что он сходится. В общем случае $\widehat{D}(x)$ и $\hat{\varepsilon}(x)$ уравнение $(48)$ описывает очень широкий спектр физических процессов, таких как диффузия, распространение тепла, эволюция пакетов заряженных частиц и т. д. Решение полностью зависит 
от явного вида операторов $\hat{\varepsilon}(x), \widehat{D}(x)$ и от значения коммутатора $\left[\hat{\varepsilon}^{2}, \widehat{D}\right]$. В нашем случае $\varepsilon=$ const и коммутатор с $\widehat{D}(x)=\alpha \partial_{x}^{2}+\beta x \partial_{x}$ равен нулю. Тогда имеем

$$
F(x, t)=e^{(-t / 2) \varepsilon} \frac{t}{4 \sqrt{\pi}} \int_{0}^{\infty} e^{-t^{2} / 16 \xi-\xi \varepsilon^{2}} e^{-a \partial_{x}^{2}-b x \partial_{x}} f(x) \frac{d \xi}{\xi \sqrt{\xi}},
$$

где $a=4 \xi \alpha>0, b=4 \xi \beta>0, a / b=\alpha / \beta$. Таким образом, экспонента $\widehat{U}=e^{-a \partial_{x}^{2}-b x \partial_{x}}$ отличается от экспоненты в решении обычного уравнения Фоккера-Планка знаком и независимостью от времени. Используя тождество

$$
e^{\hat{A}+\hat{B}}=e^{\hat{A}\left(1-e^{-m}\right) / m} e^{\hat{B}}
$$

справедливое при $[\hat{A}, \hat{B}]=m \hat{A}$ (в нашем случае $\hat{A}=-a \partial_{x}^{2}, \hat{B}=-b x \partial_{x}, m=-2 b$ ) и соотношение

$$
e^{-b x \partial_{x}} f(x)=f\left(e^{-b} x\right),
$$

согласно работам [19] и [21] имеем $\widehat{U} f(x)=\widehat{S} f(y)$, где $\widehat{S}=e^{(-\rho / 2) \partial_{y}^{2}}, y=e^{-b} x$. Итак, искомое решение уравнения (47) преобразуется к интегралу от действия оператора $\widehat{S}=e^{(-\rho / 2) \partial_{y}^{2}}$ на функцию $f(y)$ :

$$
F(x, t)=\frac{t e^{-t \varepsilon / 2}}{4 \sqrt{\pi}} \int_{0}^{\infty} e^{-t^{2} / 16 \xi-\xi \varepsilon^{2}} \widehat{S} f(y) \frac{d \xi}{\xi \sqrt{\xi}} .
$$

Выберем в качестве начальной функцию Гаусса $f(x)=e^{-x^{2}}$, под действием оператора эволюции преобразующуюся в

$$
\widehat{U} f(x)=\widehat{S} f(y)=\frac{1}{\sqrt{1-2 \rho}} \exp \left[-\frac{e^{-2 b} x^{2}}{1-2 \rho}\right],
$$

где $\rho=(a / b)\left(1-e^{-2 b}\right)=(\alpha / \beta)\left(1-e^{-8 \beta \xi}\right)$. Таким образом, эволюция начального распределения Гаусса $f(x)=e^{-x^{2}}$ при условии ограниченности решения уравнения (47) определяется выражением

$$
\begin{gathered}
\left.F(x, t)\right|_{f(x)}=e^{-x^{2}}=\frac{t e^{-\varepsilon t / 2}}{4 \sqrt{\pi}} \int_{0}^{\infty} \exp \left(-\frac{t^{2}}{16 \xi}-\xi \varepsilon^{2}-\frac{e^{-8 \beta \xi} x^{2}}{1-2 \alpha\left(1-e^{-8 \beta \xi}\right) / \beta}\right) \times \\
\times \frac{d \xi}{\xi \sqrt{\xi} \sqrt{1-2 \alpha\left(1-e^{-8 \xi \beta}\right) / \beta}} .
\end{gathered}
$$

Уравнение (47) можно также записать в виде

$$
\left(\tau \partial_{t}^{2}+\partial_{t}\right) F(x, t)=\left(A \partial_{x}^{2}+B x \partial_{x}\right) F(x, t),
$$

где $\tau=1 / \varepsilon, A=\alpha / \varepsilon, B=\beta / \varepsilon$. Графики полученного решения (54) представлены на рис. 5-7.

Отметим быстрое затухание решения при малых значениях параметра $\tau$, т. е. при больших $\varepsilon$ (ср. рис. 5 и рис. 7). Решение симметрично по $x$, а вклад слагаемого с $\beta \neq 0$ просматривается в общем подъеме и выпуклости (т. е. знаке производной $\left.\partial_{t}^{2} F\right)$ построенного решения по $t$ при $t>0$ независимо от значения $x$ 


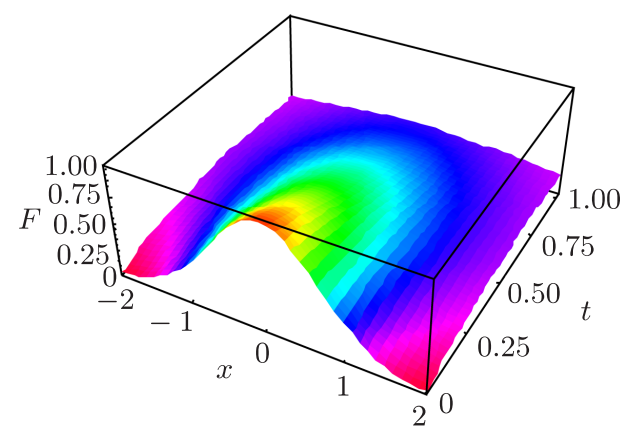

Рис. 5. Пример графика решения обобщенного уравнения Фоккера-Планка $\left(\tau \partial_{t}^{2}+\partial_{t}\right) F=\left(A \partial_{x}^{2}+B x \partial_{x}\right) F$ для $F(x, 0)=e^{-x^{2}}, F(x, \infty)<\infty$ при $\tau=2 / 3$, $B=8 / 3(\alpha=1, \beta=4, \varepsilon=1.5)$.

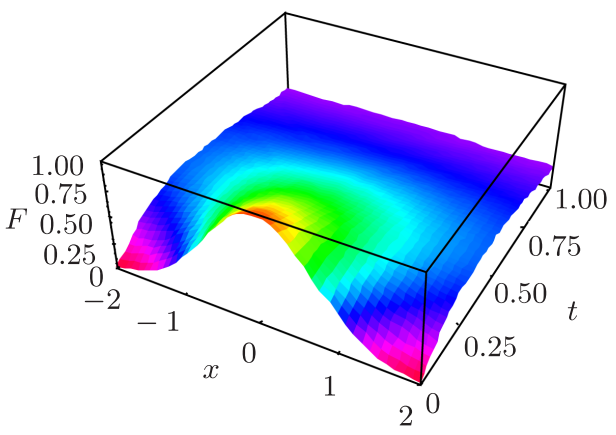

Рис. 6. Пример графика решения обобщенного уравнения Фоккера-Планка $\left(\tau \partial_{t}^{2}+\partial_{t}\right) F=\left(A \partial_{x}^{2}+B x \partial_{x}\right) F$ для $F(x, 0)=e^{-x^{2}}, F(x, \infty)<\infty$ при $\tau=2 / 3$, $A=2 / 3, B=32 / 3(\alpha=1, \beta=16, \varepsilon=1.5)$.

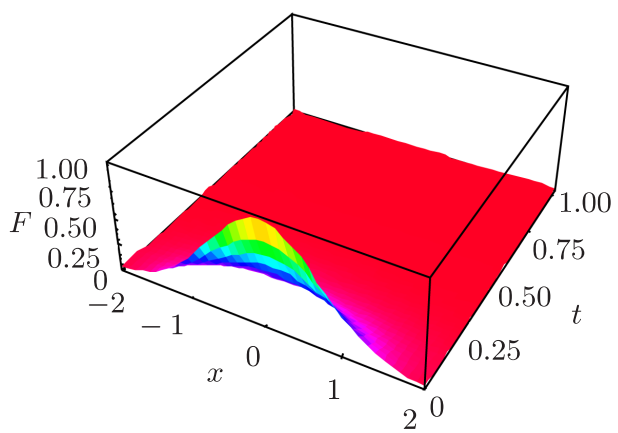

Рис. 7. Пример графика решения обобщенного уравнения Фоккера-Планка $\left(\tau \partial_{t}^{2}+\partial_{t}\right) F=\left(A \partial_{x}^{2}+B x \partial_{x}\right) F$ для $F(x, 0)=e^{-x^{2}}, F(x, \infty)<\infty$ при $\tau=1 / 15$, $A=2 / 3, B=8 / 3$ ( $\alpha=10, \beta=40, \varepsilon=15)$. 


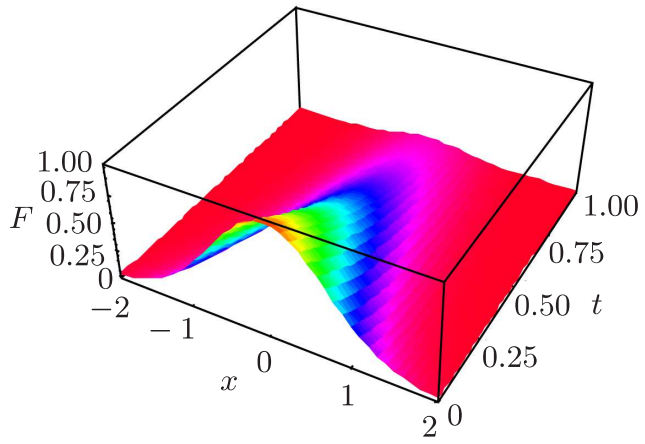

Рис. 8. Пример графика решения обычного уравнения Фоккера-Планка $\partial_{t} F=\left(A \partial_{x}^{2}+B x \partial_{x}\right) F, F(x, 0)=e^{-x^{2}}$ для $F(x, \infty)<\infty$ при $A=2 / 3, B=8 / 3$.

(см. рис. 6) по сравнению с решением обычного уравнения Фоккера-Планка $\partial_{t} F=$ $\left(A \partial_{x}^{2}+B x \partial_{x}\right) F$, изображенным на рис. 8 (ср. с рис. 5$)$.

Отметим, что при малых значениях параметра $\beta$ построенное решение расширенного уравнения Фоккера-Планка (47) близко к решению гиперболического уравнения теплопроводности Каттанео [37], которое получается при $\beta=0$ и качественно описывает такие явления, как второй звук в жидком гелии [38] и в твердых кристаллах [39], теплопроводность при низких температурах менее $25 \mathrm{~K}$ и другие явления.

Для контраста приведем операторное решение похожего на (47) уравнения, включающего смешанную производную по координате и времени:

$$
\left(\frac{\partial^{2}}{\partial t^{2}}+\varepsilon \frac{\partial^{2}}{\partial x \partial t}\right) F(x, t)=\left(\frac{\partial^{2}}{\partial x^{2}}+\beta x \partial_{x}\right) F(x, t), \quad \varepsilon, \beta=\mathrm{const}
$$

которое содержит оператор $\hat{\varepsilon}=\varepsilon \partial_{x}$, коммутирующий со второй производной по координате в $\widehat{D}$. Действуя по аналогии с решением уравнения типа Фоккера-Планка (47), получаем равенство

$$
F(x, t)=\frac{t}{4 \sqrt{\pi}} \int_{0}^{\infty} e^{-t^{2} / 16 \xi} \widehat{\Theta} \widehat{S} f(y) \frac{d \xi}{\xi \sqrt{\xi}},
$$

где

$$
\begin{gathered}
\widehat{S}=e^{-(\rho / 2) \partial_{y}^{2}}, \quad \widehat{\Theta}=e^{(-t / 2) \varepsilon \partial_{x}} \\
\rho=\frac{a}{b}\left(1-e^{-2 b}\left(1-\xi \varepsilon^{2} \frac{2 b}{a}\right)\right)=\frac{\alpha}{\beta}\left(1-e^{-8 \beta \xi}\left(1-\xi \varepsilon^{2} \frac{2 \beta}{\alpha}\right)\right) .
\end{gathered}
$$

Для уравнения с начальным условием в виде функции Гаусса $f(x)=e^{-x^{2}}$ получаем ограниченное при $t=\infty$ решение следующего вида:

$$
\begin{aligned}
\left.F(x, t)\right|_{f(x)=e^{-x^{2}}}= & \frac{t}{4 \sqrt{\pi}} \int_{0}^{\infty} \exp \left(-\frac{t^{2}}{16 \xi}-\frac{e^{-8 \xi \beta}(x-t \varepsilon / 2)^{2}}{1-2 \alpha\left(1-e^{-8 \beta \xi}\left(1-\xi \varepsilon^{2} 2 \beta / \alpha\right)\right) / \beta}\right) \times \\
& \times \frac{d \xi}{\xi \sqrt{\xi} \sqrt{1-2 \alpha\left(1-e^{-8 \beta \xi}\left(1-\xi \varepsilon^{2} 2 \beta / \alpha\right)\right) / \beta}} .
\end{aligned}
$$




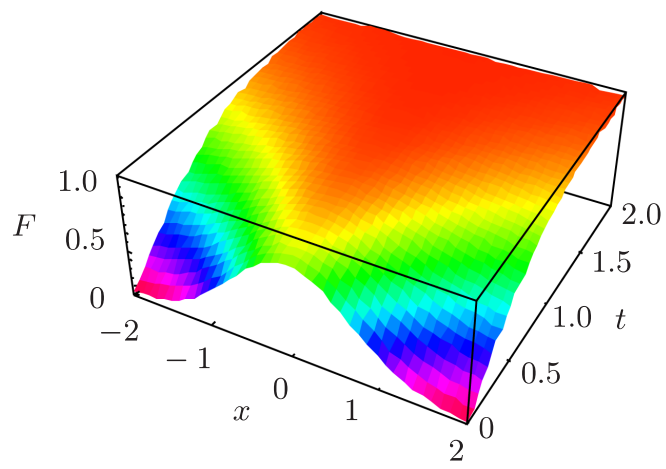

Рис. 9. Пример графика ограниченного решения уравнения $\left(\partial_{t}^{2}+\varepsilon \partial_{x, t}^{2}\right) F(x, t)=\left(\alpha \partial_{x}^{2}+\beta x \partial_{x}\right) F(x, t)$ для начальной функции $f(x)=e^{-x^{2}}$ при $\alpha=1, \beta=5, \varepsilon=0$.

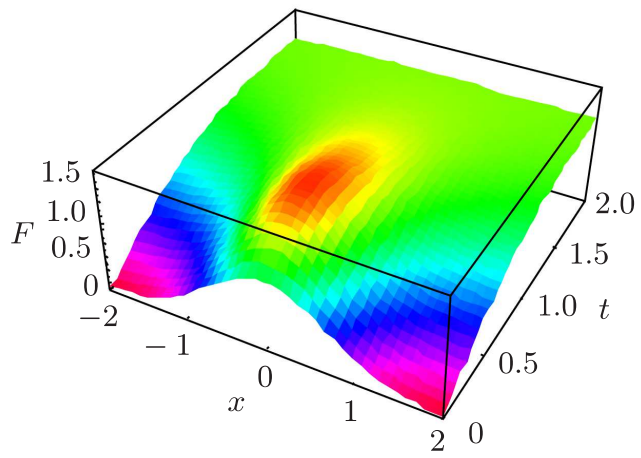

Рис. 10. Пример графика ограниченного решения уравнения $\left(\partial_{t}^{2}+\varepsilon \partial_{x, t}^{2}\right) F(x, t)=\left(\alpha \partial_{x}^{2}+\beta x \partial_{x}\right) F(x, t)$ для начальной функции $f(x)=e^{-x^{2}}$ при $\alpha=1, \beta=5, \varepsilon=4$.

Для $\varepsilon=0$ результат упрощается до выражения

$$
\begin{aligned}
\left.F(x, t ; \varepsilon=0)\right|_{f(x)=e^{-x^{2}}=} & \frac{t}{4 \sqrt{\pi}} \int_{0}^{\infty} \exp \left(-\frac{t^{2}}{16 \xi}-\frac{e^{-8 \xi \beta} x^{2}}{1-2 \alpha\left(1-e^{-8 \xi \beta}\right) / \beta}\right) \times \\
& \times \frac{d \xi}{\xi \sqrt{\xi} \sqrt{1-2 \alpha\left(1-e^{-8 \xi \beta}\right) / \beta}} .
\end{aligned}
$$

Функция $\left.F(x, t)\right|_{f(x)=e^{-x^{2}}}$ при $\alpha=1, \beta=5, \varepsilon=0$ построена на рис. 9. Решение растет со временем и достигает насыщения при $t>2$, в отличие от решения (54) уравнения (47), которое затухает. Пример решения для $\alpha=1, \beta=5, \varepsilon=4$ приведен на рис. 10. Ненулевые значения $\varepsilon$ нарушают монотонность роста и приводят к появлению локального максимума (см. рис. 10). В обоих случаях принцип максимума не выполняется, как видно из рис. 9 и из рис. 10. 


\section{7. ВЫВОДЫ}

С помощью операторного метода получены решения для различных классов дифференциальных уравнений. При этом использовались обратные дифференциальные операторы и экспоненциальные операторы, широко применялись операторные тождества и интегральные преобразования. В частности, получено операторное решение обыкновенного дифференциального уравнения с производной действительного нецелого порядка в виде свертки, включающей функцию Макдональда (Бесселя второго рода мнимого аргумента). Получено операторное решение расширенного уравнения типа Блэка-Шоулза с дополнительными слагаемыми. Решено уравнение типа Блэка-Шоулза с производной Лагерра, т. е. уравнение с производными четвертого порядка по координате.

Построено решение уравнения Шредингера для заряда в электрическом поле при прохождении потенциального барьера в двумерном случае. Квантовомеханическая интерпретация полученного решения уравнения Шредингера для частицы в электростатическом поле представляет собой амплитуду вероятности того, что частица, находившаяся в начальный момент времени в исходной точке $x=0$, за время $t$ перейдет в точку с координатой $x$. Для произвольной функции начального условия $f$ решение записывается в виде ее свертки с решением для начальной $\delta$-функции Дирака. Построено решение расширенного уравнения теплопроводности Фурье и продемонстрировано распространение $\delta$-образного импульса, моделирующего наиболее широко распространенную технику экспериментального измерения теплопроводности с помощью сверхкороткого начального лазерного импульса. Получены точные аналитические решения уравнения теплопроводности Фурье в двумерном случае. Проанализированы распространение начальной $\delta$-функции и экспоненциально-степенной функции, позволяющие моделировать практически любые виды импульсов.

Решены операторным методом различные разновидности уравнения ФоккераПланка, моделирующие распространение пучков электронов, в частности, для лазеров на свободных электронах. Приведено графическое сравнение полученных решений друг с другом. Все решения получены точно и в явном аналитическом виде. Принцип максимума, который обеспечивает единственность и устойчивость решения, выполняется для уравнения теплопроводности Фурье только при отсутствии дополнительных членов в уравнении. Из рассмотренных нами примеров уравнений этого типа принцип максимума не выполнен для решений уравнений (25), (28), (31) при наличии линейных и других членов, поскольку максимум решения в пространственно-временно́м прямоугольнике $R=\{0 \leqslant x \leqslant 1,0 \leqslant t \leqslant T\}$ достигается не в начальный момент $(t=0)$ и не при крайних значениях координат $(x=0$ или $x=1)$. Для уравнения Фоккера-Планка (56) принцип максимума также не соблюдается. Последнее возможно, так как рассмотренное уравнение не является ни эллиптическим, ни параболическим. При получении решений нами использовались обобщенные формы полиномов Лагерра и Эрмита, что позволило написать их в виде разложения в ряды по полиномам указанных выше типов. Использование при этом операторных определений и представлений позволило легко применить их для решения математических задач и проблем, возникающих при моделировании физических процессов распространения тепла, пучков частиц и зарядов в рамках классической и квантовой механики. 
Показано, что инвертирование дифференциальных операторов и применение обратной производной часто открывает путь к непосредственному получению аналитических решений и позволяет продвинуться в решении сложных математических проблем и связанных с ними физических процессов. Проведенное исследование показало, что операторный подход в сочетании с интегральными преобразованиями, использованием расширенных форм ортогональных полиномов и специальных функций и операторных соотношений представляет собой мощный инструмент исследования широкого спектра физических проблем.

Развитая выше операторная техника применима к решению и других дифференциальных уравнений, описывающих широкий спектр различных физических процессов. Они будут рассмотрены в следующих публикациях.

\section{Список литературы}

[1] V. I. Denisov, B. N. Shvilkin, V.A. Sokolov, M. I. Vasili'ev, "Pulsar radiation in postMaxwellian vacuum nonlinear electrodynamics", Phys. Rev. D, 94:4 (2016), 045021, 6 c.

[2] В. И. Денисов, А. В. Козарь, В.Ф. Шарихин, "Исследование траекторий намагниченной частицы в плоскости экватора магнитного диполя", Вестн. Моск. ун-та. Сер. 3. Физ. Астрон., 3 (2010), 8-13.

[3] V. M. Pastukhov, Y. V. Vladimirova, V. N. Zadkov, "Photon-number statistics from resonance fluorescence of a two-level atom near a plasmonic nanoparticle", Phys. Rev. A, 90:6 (2014), 063831, 11 pp.

[4] G. Dattoli, V. V. Mikhailin, K. V. Zhukovsky, J. Appl. Phys., 104:12 (2008), 124507, 8 pp.

[5] Д. Даттоли, К. В. Жуковский, В. В. Михайлин, "Влияние постоянного магнитного поля на излучение плоского ондулятора", Вестн. Моск. ун-та. Сер. 3. Физ. Астрон., 5 (2009), 33-38.

[6] K. V. Zhukovsky, "High harmonic generation in the undulators for free electron lasers", Opt. Commun., 353 (2015), 35-41.

[7] K. V. Zhukovsky, "Analytical account for a planar undulator performance in a constant magnetic field", J. Electromagn. Waves Appl., 28:15 (2014), 1869-1887.

[8] K. V. Zhukovsky, "Harmonic generation by ultrarelativistic electrons in a planar undulator and the emission-line broadening", J. Electromagn. Waves Appl., 29:1 (2015), 132-142.

[9] V. I. Denisov, V. A. Sokolov, M. I. Vasili'ev, "Nonlinear vacuum electrodynamics birefringence effect in a pulsar's strong magnetic field", Phys. Rev. D, 90:2 (2014), 023011, 7 pp.

[10] K. V. Zhukovsky, "Emission and tuning of harmonics in a planar two-frequency undulator with account for broadening", Laser Part. Beams, 34:3 (2016), 447-456.

[11] K. V. Zhukovsky, "Violation of the maximum principle and negative solutions with pulse propagation in Guyer-Krumhansl model", Internat. J. Heat Mass Transfer, 98 (2016), $523-529$.

[12] K. V. Zhukovsky, "Exact solution of Guyer-Krumhansl type heat equation by operational method", Internat. J. Heat Mass Transfer, 96 (2016), 132-144.

[13] Y. Zhang, W. Ye, "Modified ballistic-diffusive equations for transient non-continuum heat conduction", Internat. J. Heat Mass Transfer, 83 (2015), 51-63.

[14] K. V. Zhukovsky, H. M. Srivastava, "Analytical solutions for heat diffusion beyond Fourier law", Appl. Math. Comp., 293 (2017), 423-437.

[15] B. Căruntu, C. Bota, "Analytical approximate solutions for a general class of nonlinear delay differential equations", Sci. World J., 2014 (2014), 631416, 6 pp.

[16] S. Hesam, A. R. Nazemi, A. Haghbin, "Analytical solution for the Fokker-Planck equation by differential transform method", Scientia Iranica, 19:4 (2012), 1140-1145.

[17] K. V. Zhukovsky, "Solution of some types of differential equations: operational calculus and inverse differential operators", Sci. World J., 2014 (2014), 454865, 8 pp. 
[18] К. В. Жуковский, “Операторное решение дифференциальных уравнений с производными нецелого порядка, уравнений Блэка-Шоулза и теплопроводности", Вестн. Моск. ун-та. Сер. 3. Физ. Астрон., 2016, № 3, 18-25.

[19] G. Dattoli, H. M. Srivastava, K. V. Zhukovsky, "Operational methods and differential equations with applications to initial-value problems", Appl. Math. Comput., 184:2 (2007), 979-1001.

[20] K. V. Zhukovsky, G. Dattoli, "Evolution of non-spreading Airy wavepackets in time dependent linear potentials", Appl. Math. Comput., 217:20 (2011), 7966-7974.

[21] К. В. Жуковский, “Метод обратных дифференциальных операторов с использованием ортогональных полиномов и специальных функций для решения некоторых типов дифференциальных уравнений и физических задач", Вестн. Моск. ун-та. Сер. 3. Физ. Астрон., 2 (2015), 19-26.

[22] G. Dattoli, H. M. Srivastava, K. V. Zhukovsky, "A new family of integral transforms and their applications", Integral Transform. Spec. Funct., 17:1 (2006), 31-37.

[23] P. Appell, J. Kampé de Fériet, Fonctions Hypergéométriques et Hypersphériques. Polynômes d'Hermite, Gauthier-Villars, Paris, 1926.

[24] D. T. Haimo, C. Markett, "A representation theory for solutions of a higher-order heat equation. I", J. Math. Anal. Appl., 168:1 (1992), 89-107.

[25] G. Dattoli, H. M. Srivastava, K. V. Zhukovsky, "Orthogonality properties of the Hermite and related polynomials", J. Comput. Appl. Math., 182:1 (2005), 165-172.

[26] А. Эрдейи, В. Магнус, Ф. Оберхеттингер, Ф. Трикоми, Высшие трансиендентнъе функиии, Наука, М., 1966.

[27] П. А. Вшивцева, В. И. Денисов, И. П. Денисова, "Интегральное соотношение для тензорных полиномов", ТМФ, 166:2 (2011), 216-224.

[28] K. B. Wolf, Integral Transforms in Science and Engineering, Mathematical Concepts and Methods in Science and Engineering, 11, Plenum Press, New York, 1979.

[29] H. M. Srivastava, H.L. Manocha, A Treatise on Generating Functions, John Wiley and Sons, New York, 1984.

[30] H. W. Gould, A. T. Hopper, "Operational formulas connected with two generalizations of Hermite polynomials", Duke Math. J., 29 (1962), 51-63.

[31] G. N. Watson, A Treatise on the Theory of Bessel Functions, Cambridge Univ. Press, Cambridge, 1944.

[32] K. V. Zhukovsky, "Operational solution for some types of second order differential equations and for relevant physical problems", J. Math. Anal. Appl., 446:1 (2017), 628-647.

[33] K. V. Zhukovsky, "Operational method of solution of linear non-integer ordinary and partial differential equations", SpringerPlus, 5 (2016), 119, 25 pp.

[34] А. А. Соколов, И. М. Тернов, В. Ч. Жууовский, А. В. Борисов, Калибровочнъе поля, Изд-во Моск. ун-та, М., 1986.

[35] W. J. Parker, R. J. Jenkins, C.P. Butler, G. L. Abbott, "Flash method of determining thermal diffusivity, heat capacity, and thermal conductivity", J. Appl. Phys., 32:9 (1961), 1679-1684.

[36] R. Kovács, P. Ván, "Generalized heat conduction in heat pulse experiments", Internat. J. Heat Mass Transfer, 83 (2015), 613-620.

[37] C. Cattaneo, "Sur une forme de l'équation de la chaleur éliminant le paradoxe d'une propagation instantanée", C. R. Acad. Sci. Paris, 247 (1958), 431-433.

[38] V. Peshkov, "Second sound' in Helium II", J. Phys., 8:6 (1944), 381.

[39] C. C. Ackerman, W. C. Overton, "Second sound in solid Helium-3", Phys. Rev. Lett., 22:15 (1969), 764-766. 